\title{
sciendo
}

\section{Mode effect analysis in the case of daily passenger mobility survey}

\author{
Vasilij Centrih \\ Statistical Office of the Republic of Slovenia, Ljubljana, Slovenia \\ vasilij.centrih@gov.si \\ Andrej Viršček \\ Statistical Office of the Republic of Slovenia, Ljubljana, Slovenia \\ andrej.virscek@gov.si \\ Andreja Smukavec \\ Statistical Office of the Republic of Slovenia, Ljubljana, Slovenia \\ andreja.smukavec@gov.si \\ Nataša Bučar \\ Statistical Office of the Republic of Slovenia, Ljubljana, Slovenia \\ natasa.bucar2@gov.si \\ Marta Arnež \\ Statistical Office of the Republic of Slovenia, Ljubljana, Slovenia \\ marta.arnez@gov.si
}

\begin{abstract}
In the autumn 2017, The Statistical Office of the Republic of Slovenia (SURS) has conducted for the first time a survey on daily passenger mobility of Slovenian residents. The key statistics are on persons' daily traveling habits, such as number of trips, travelled distance, time spent on traveling, and so on. Two independent samples were selected for the simultaneous collection of data by two modes, faceto-face interview (CAPI) and online questionnaire (WEB). The goal of this study is to identify the possible sources of mode measurement errors, with the objective to better design and thus improve the whole data collection process. The detailed mode effect analysis is performed by the comparison of the key statistic estimates and the use of regression models. Usually the measurement mode effect is an issue in surveys on the more sensitive topics or persons' opinions. This work points out that, first, the mode measurement effect can be an issue also in a more factual survey content, and second, the corresponding statistical data processes can have an important contribution to minimising measurement errors. The results show that WEB respondents are inclined to join two or more trips into one reported, which gives lower estimate of average number of daily trips. The main reason is the demanding questionnaire content. Additionally, the complex data editing process was still insufficient to correct completely for such measurement error. The possible improvements of the data collection process are also discussed.
\end{abstract}

Keywords: data comparability, mixed mode surveys, mode measurement effect, mode selection effect. 
JEL classification: C10, C18, C83, R41.

DOI: 10.2478/crebss-2020-0010

Received: October 30, 2020

Accepted: November 30, 2020

\section{Introduction}

In recent years an online (WEB) data collection mode has become a relevant and reliable tool of survey data collection and a regular praxis also in official statistics. Since 2016 several household surveys at the Statistical Office of the Republic of Slovenia (SURS) has been transferred to the WEB survey mode (Arnež et al., 2018). Transition to WEB has opened several methodological challenges, such as coverage, non-response and possible measurement issues (Bučar et al., 2018). Most commonly the WEB mode is used in combination with additional mode, usually face-to-face interview (CAPI). Because the main advantage of WEB is cost effectiveness, it is usually used as a first collection mode and the CAPI collection mode is used subsequently for WEB non-respondents.

In the autumn 2017, SURS has conducted a Survey on daily passenger mobility, which examines persons' daily traveling habits, such as number of daily trips, duration and distance of trips, purpose of traveling (work, education, leisure, shopping, etc.), transport modes (walking, cycling, public transport, car, etc.), and so on (Škafar, 2018). The survey was supported by Eurostat and has followed its guidelines on passenger mobility statistics, which aim at a development of methodology for a harmonised data collection in EU (Eurostat, 2018). However, specificity of our survey is the two independent samples that were selected for the simultaneous collection of data by two modes, CAPI and WEB. When examining some statistic estimates separately for each mode in our survey, we noticed that some estimates quite differ between mode, while some others are almost identical. The noticeable difference was observed in the declared number of trips per person in a day. Namely, the WEB respondents reported a bit less trips. The independent mode samples in our (concurrent) survey open a specific possibility to study the mode effect. The analysis with regression models can be more straightforward and less complex than in sequential mixed-mode surveys (Berzelak, 2014; Klaush, 2014).

The objective of this study is first, to find out if the observed estimate differences can be attributed to the so-called mode effect, and second, to identify all possible sources of mode measurement errors. Namely, usually the measurement mode effect is an issue in surveys on the more sensitive topics or persons' opinions. Our analysis shows that, first, the mode measurement effect can be an issue also in a more factual survey content, and second, the corresponding statistical data processes can have an important contribution to minimising measurement errors. By carefully identifying the measurement error propagation, we can better design and thus improve the whole data collection process in official statistics.

The theoretical background needed to tackle the mode effect is reviewed in the following Literature review section. The overview of similar analysis and results in the field of mobility and transport surveys is also given. After that, the Research methodology section presents the data collection process. The findings from questionnaire design stage as well as from the data editing processes are also presented, and the methodology for mode effect analysis is set up. The Results and discussion section presents the results on response rate, the lessons learned from the process of data clearing and correction, comparison of key statistic estimates, the 
corresponding regression analysis of mode measurement effect in key statistic estimates which is followed by discussion.

\section{Literature Review}

In this study, we rely on the conceptualisation of the mode effect as defined by de Leeuw (2018). It distinguishes between the mode selection effect and the mode measurement effect, where the net effect of the two components forms the socalled mode effect. The mode selection effect, defined also as the differential nonobservation error, pertains to the difference in response (who responds and who does not in which mode). On the other hand, the mode measurement effect, defined also as the differential observation error, pertains to the fact that same person answers differently in different modes. Mode selection effect in a mixedmode study can be a desired effect, because it reduces the coverage and nonresponse errors of single mode designs, and it is one of the main reasons why mixed-mode approaches are implemented. On the other hand, mode measurement effect is a source of unwanted measurement error. Usually the measurement error is an issue in surveys on the more sensitive topics or persons' opinions. The error sources can be social desirability, acquiescence, extremeness, selection of middle scale values, recency effect, primacy effect, etc. (de Leeuw, 2018). In our case, the main potential sources for measurement effect lie in the socalled cognitive burden, questionnaire design and some other more technical issues regarding the data collection process.

To be able to reduce these errors, the researches should carefully design the total mixed-mode survey process from the beginning: reduction of the measuring effect by carefully designing the questionnaire, use of auxiliary data to estimate the measurement error, etc. The mode measurement effect can be measured after the correction for the mode selection effect by controlling differences between modes regarding the auxiliary data comprised at least of socio-demographic variables (Hox, de Leeuw, Klausch, 2017). The mode effect is usually the most pronounced between the two distinct collection types (Klausch, 2014), i.e. the interview (which is CAPI in our case) and the self-administered mode (which is WEB in our case).

Regarding the content, a similar mobility survey to ours was a French household travel survey (Bayart, Bonnel, 2010; 2015). The study was performed in a sequential mixed-mode: CATI (telephone interview) first and then WEB, offered to CATI nonrespondents). The WEB respondents' characteristics are found to be similar to our experiences (Bučar et al., 2018): WEB respondents are younger, more educated, their most frequent activity statuses are employed or students, and so on. They have observed the similar potential measurement effect in the number of declared trips in a day. Namely, the WEB respondents report les trips per day than CAPI respondents (Bonnel, Bayart, Smith, 2015). Detailed analysis showed that WEB respondents report significantly less short walking trips or less constrained trips. It is possible, that WEB respondents simply omit the 'not-so-important' trips. Additionally, the main potential problem is identified: respondents do not always find it easy to understand or absorb the concept of trip (Bayart, Bonnel, 2015). Some other similar surveys may be found, performed also on a regular basis by some European countries, e.g. Netherlands (Statistics Netherlands, 2016), Sweden (Transport Analysis, 2019) and Denmark (Center for Transport Analytics, 2019).

Regarding the methodology, an elaborate analysis on mode effect, where independent mode samples (CATI, CAPI and WEB) have been used, was performed by Berzelak (2014). Mode measurement effect was studied with OLS, logit and ordered logit (partial proportional odds) regression models. The focus was mainly on 
opinion statistics - scale questions with multiple choice answers. All effects were controlled for basic socio-demographic structures (gender, age, education). The study also suggests the frequency of internet usage as an additional auxiliary variable for WEB mode effect analyses. Namely, some studies reported smaller difference between modes among more frequent internet users (Berzelak, 2014). As opposed to the complex sequential mixed-mode surveys, the analysis of the mode measurement effect with independent samples can be directly addressed by regression models, where besides auxiliary variables, the mode is included as an additional covariate (Martin, Lynn, 2011).

\section{Research Methodology \\ Data}

The basis for the sampling frame of our survey was the Central Population Register and the corresponding Demographic Database prepared by SURS. The sampling frame was the population of Slovenia aged 15-84 in the period of data collection and living in private households. The sample for WEB was one-stage stratified systematic and the sample for CAPI was two-stage stratified. Stratification was geographical (12 statistical regions) and by type of settlement (6 types according to the size of settlement and a share of farms within the settlement). 15,015 people aged 15-64 were selected in the WEB sample, and 8,001 people aged 15-84 were selected for CAPI (Škafar, 2019a). Because the population aged 65-84 was not included in the WEB sample, only the population aged 15-64 was considered from both modes, for the purpose of our mode analysis in the presented study. That gives us a combined sample of 20,365 persons (5,350 CAPI and 15,015 WEB). The process of data weighting included the sampling design, the unit nonresponse rate, and the calibration on the available auxiliary variables (sex, age, education, activity status, statistical regions and type of settlement). The two independent samples were weighted separately. To create a single estimator the two samples were combined using the additional weight factor that considered sample size proportions.

The content of the WEB and CAPI questionnaires was the same, some differences were in wording where necessary. The questionnaire comprises of (1) questions about the respondent, the selected day and possible reasons for not making any trip that day, (2) trips and stages (parts) of the trip (if several means of transport were used) and (3) questions about household, use of public transport and carpooling, ownership of (electric) bicycles, and so on. A definition of a trip is the following: the movement on a public road, path made for a certain purpose such as work, school, shopping, etc. A trip-maker could walk or use one or more modes of transport. Only trips longer than $100 \mathrm{~m}$ and up to $300 \mathrm{~km}$ were taken into account. The trip could start or end over the country border, and the trips taking place fully abroad were excluded. A trip could also be a loop, e.g. Walk, recreational running.

For the survey, also the information/motivation letter has been prepared. Besides the standardised information on the survey, the information letter motivates the selected person to take part in the survey and, most importantly, explains the subject of the survey - in writing and drawings. The letter included two graphical models of a day with different trips and stages. This part of the letter also has a role of a memory jogger to lessen the impact of memory recall effect. The selected person could have thought about his/her trips during the selected day (especially in WEB mode). In the pilot survey, it was confirmed that the content of the survey is quite demanding. We found out, that the understanding of the concept of a trip (definition, start, end, purpose, 1 purpose means 1 trip, 1 transport mode means 1 stage of a trip, etc.) is 
the crucial point of the survey. Both, the respondents and the interviewers reported that the information letter was very useful and informative.

\section{Methods}

The number of final completed questionnaires in sample with population aged 15-64 is 6,974. However, in the analysis of key statistic estimates, we focus on the so-called trip-makers, i.e. persons that made at least one trip on the selected day. Share of trip-makers is $87.7 \%$ in CAPI and $88.9 \%$ in WEB mode (on weighted data). A detailed analysis showed that the differences in the shares of trip-makers between modes are significantly small and does not differ much even across other controlling variables. The sample of the responses with the trip-makers aged 15-64, comprises 6,216 persons (2,642 CAPI and 3,574 WEB).

First, we examine the response rate by basic socio-demographic variables and discuss the differences between two modes (mode selection effect). Second, we review the process of data editing and try to identify the possible sources of mode measurement error. Third, we compare the key statistic estimates broken-down by basic socio-demographic variables and by some auxiliary variables. We focus on the two most indicative statistics: average number of trips per mobile person in a day and the total distance travelled by one person in a day. By graphical representation of the estimates, we get a sense of possible differences due to mode.

In the end, we apply the regression models to two key statistic estimates to study the mode measurement effect. The number of trips in a day is a count data variable, therefore, we use a dispersed Poisson model for its distribution within the generalised linear model. On the other hand, the daily distance is the sum of individual trip distances. Despite the fact, that the daily distance is associated with the number of trips and has thus heavier right tail, it is not a count data. Therefore, we use an OLS regression model on the logarithm of daily distance. Since we have combined independent samples, we analyse the mode measurement effect by including the mode in the regression model as an independent covariate. We control the regression for basic socio-demographic variables and some auxiliary variables.

\section{Results and Discussion Response Rate and Mode Selection Effect}

The overall response rate was $38.4 \%$, where the response rate for CAPI mode was $60.9 \%$ and for WEB mode $26.3 \%$. Based on experiences we expected approximately $60 \%$ response rate for CAPI and $30 \%$ for WEB.

The comparison of the response rates by basic socio-demographic categories (sex, age, education and activity status) is presented in diagrams in figure 1. In all diagrams, the overall response rates for CAPI (blue) and WEB (red) modes are depicted with dashed lines, marking the considerable difference between modes. We can see, that women are in general a bit better respondents than men. Age group 56-65 has highest response in CAPI mode and lowest in WEB. Significant higher response has the population with post-secondary education in WEB, whereas the response in CAPI slightly decreases with education level. Special activity group are Retired with the highest response in CAPI and almost the lowest in WEB. In the WEB, we can confirm the considerably higher response rate than the WEB average in traditionally known socio-demographic groups, such as younger respondents, higher educated and students (Bučar et al., 2018). The corresponding WEB response rates for these groups are: younger aged $15-25$ with $33.2 \%$, post-secondary education with $40.5 \%$ and pupils and students with $37.1 \%$. 
The mode selection effect can be present, if statistic estimates differ between the above socio-demographic groups. For example, if higher educated population makes more trips and the same group has higher response rate in WEB mode, the estimate in WEB mode will give larger number of daily trips in total. Weight calibration that includes education corrects for this effect. Namely, by weight calibration we approximate the respondents' structure to the population structure, and therefore minimise the so-called mode selection effect in the statistic estimates. The assumption here is that the respondents and non-respondents do not differ by some other not considered characteristic, which would be at the same time associated with the traveling behaviour. The latter would give us the bias due to non-response.

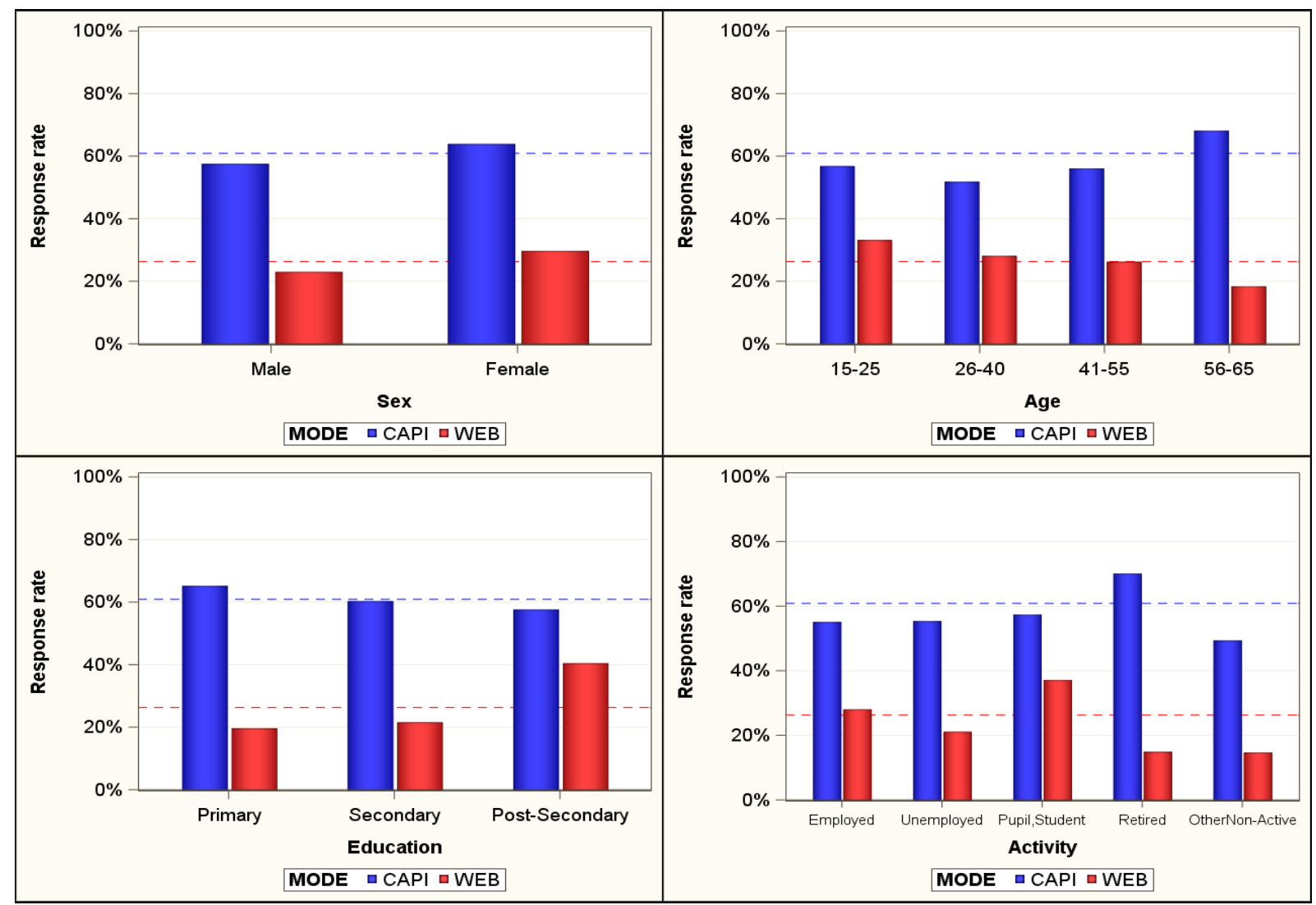

Figure 1 Response rate by mode, broken down by basic socio-demographic variables: sex (upper left), age (upper right), education (lower left) and activity status (lower right). Source: SURS, 2020.

\section{Data Editing}

Data were statistically edited with the combination of systematic corrections and imputation procedures. The most frequent corrections were made because of illogical ratio between the trip distance and duration according to the means of transport. The records on distance and duration were most frequently imputed, especially for trip stages. We also found out that especially WEB respondents were inclined to join two trips, e.g. trip to work and trip back home were joined into one trip. This was identified by comparing the starting and ending locations, the trip distance and the purpose of a trip. We presume that in all such cases, the geocoding would be of great help, which is one of the major lessons learned. In the end, besides the planned systematic corrections, many manual corrections were necessary (Škafar, 2018; 2019b). 
Table 1 compares the editing rate by mode for 3 key statistics on the data with population aged 15-64. In the table, we can see that statistical editing was substantially higher in WEB mode. Detailed examination showed that statistical corrections are quite uniformly distributed across basic socio-demographic variables (sex, age, education and activity status). The most important differences are between modes and between different statistics, where the latter partly corresponds to the editing methodology decisions. However, although a bit high, the editing rate difference between modes is nevertheless expected. Based on experiences, there are usually a lot more mistakes done in the case of self-completion modes (WEB mode in our case), especially when the questionnaires are more demanding (Klausch, 2014).

Table 1 Editing rate by mode for 3 key statistics

\begin{tabular}{|l|c|c|c|}
\hline \multicolumn{1}{|c|}{ Statistic } & Both modes & CAPI & WEB \\
\hline Number of Trips & $7.0 \%$ & $3.2 \%$ & $9.9 \%$ \\
\hline Trip Distance & $13.9 \%$ & $6.8 \%$ & $19.2 \%$ \\
\hline Trip Time & $22.3 \%$ & $12.5 \%$ & $29.8 \%$ \\
\hline
\end{tabular}

Source: SURS, 2020.

The presented experiences in data editing process imply that the main source of the possible measurement error might be the combination of the two issues. First is the demanding content of questionnaire and the consequential cognitive burden on respondents especially when completing WEB questionnaire (Škafar, 2019b). WEB respondents might have not understood the questions correctly or completely. There is also a possibility that some wanted to complete the questionnaire more quickly (e.g. if they did many trips in a day, the motivation to fill in all trips may drop). Second, there is however the possibility of still persisting imperfections in data collection (especially in WEB) and editing techniques (missing complex controls and connections, geocoding), and the consequential significant burden on data editing processes, which could not correct $100 \%$ for all imperfections.

\section{Key Statistic Estimates Comparison}

We compare the two most indicative statistic estimates: (1) the average number of trips per trip-maker in a day, and (2) the daily travelled distance per trip-maker in a day (sum of all trip distances in a person's day). The calculated estimates are presented for basic socio-demographic variables (sex, age, education and activity status) and for some auxiliary variables provided by Central Population Register (degree of urbanisation) or by the questionnaire (salary level and number of children under age 15 in a household). A degree of urbanisation is a category with 3 levels and it was chosen due to its resemblance to two other categories considered in the weighting process: statistical region and type of settlement. Continuing with one category (degree of urbanisation) simplifies the analysis while preserving the main effect of geographical differences.

\section{Number of Trips}

The overall estimates of the average number of trips are compared in table 2 and the details are examined in figure 2. The table compares the combined sample estimate (Both Modes) and estimates by each sample mode separately. We can see that there is noticeable difference between the modes, where the CAPI mode gives larger average number of trips (3.30) than WEB mode (3.11). The overall estimates for CAPI (blue) and WEB (red) modes are marked in all diagrams in figure 2 
with dashed lines for easier comparison. The estimate values are depicted as vertical bars with 95\% confidence level limits.

Table 2 Estimates of the Number of trips by mode

\begin{tabular}{|c|c|c|c|}
\hline & Both Modes & CAPI & WEB \\
\hline Estimate & 3.189 & 3.296 & 3.109 \\
\hline Standard error & 0.023 & 0.036 & 0.031 \\
\hline
\end{tabular}

Source: SURS, 2020.

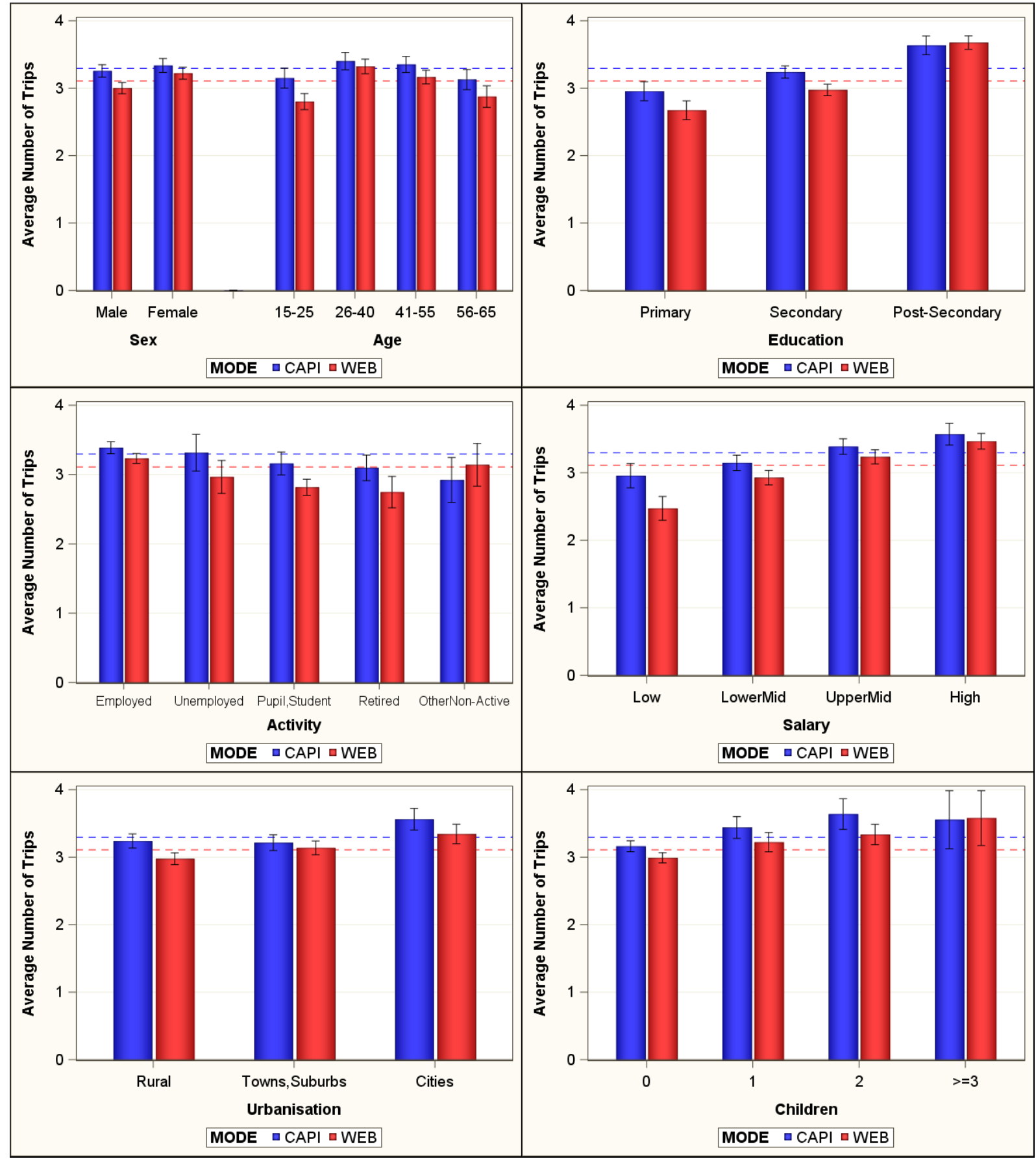

Figure 2 Average number of trips per person by: sex, age (upper left), education (upper right), activity status (middle left), salary level (middle right), degree of urbanisation (lower left) and number of children in a household (lower right). Source: SURS, 2020. 
We can see in figure 2, that in most depicted groups, the WEB estimate gives more or less lower average number of trips. There are only a few exceptions where the WEB estimate is slightly larger: post-secondary education, other non-active status and the group with 3 or more children. There are some groups with substantial lower estimates in WEB considering also the confidence level limits, which do not overlap: men, 15-25 age, secondary education, pupils and students, low salary, rural urbanisation type and the group with no children. We can be quite sure that in these groups the mode measurement effect can be present.

Beside the mode differences, some interesting trends may be observed within the categories in figure 2, such as, increasing number of trips with education, with salary level, with urbanisation and with number of children. There are less trips of group aged 15-25, and a decreasing trend with age starting from age group 26-40.

\section{Daily Distance}

The overall estimates of the daily travelled distance are compared in table 3 and the details are examined in figure 3. Table 3 compares the combined sample estimate (Both Modes) and estimates by each sample mode separately. We can see that there is a negligible difference between the modes, i.e. the CAPI mode gives $43.92 \mathrm{~km}$ and WEB mode $43.90 \mathrm{~km}$. Again, the overall estimates for CAPI (blue) and WEB (red) modes are marked in all diagrams in figure 3 with dashed lines, which practically overlap in this case. The estimate values are depicted as vertical bars with $95 \%$ confidence level limits.

Table 3 Estimates of the Daily distance $(\mathrm{km})$ by mode

\begin{tabular}{|c|c|c|c|}
\hline & Both Modes & CAPI & WEB \\
\hline Estimate & 43.91 & 43.92 & 43.90 \\
\hline Standard error & 0.78 & 1.16 & 1.05 \\
\hline
\end{tabular}

Source: SURS, 2020.

We can see in figure 3, that the estimates of daily distance quite differ between different depicted groups within each category (e.g. education). However, as opposed to the number of trips (figure 2), the comparison of daily distance estimates between modes in all individual groups shows, that the estimates do not differ substantially. Namely, practically all depicted confidence level limits overlap between modes, which implies, that there is probably no significant additional measurement effect between modes for the daily distance statistic.

Some interesting trends may be observed also in figure 3. The daily travelled distance (compared within the category) are quite shorter for women, age group 56-65 and retired persons. The daily distance increases significantly with education, and salary level, it is high for employed and pupils and students, and it decreases with the degree of urbanisation. The number of children does not have significant influence on daily travelled distance. Particularly interesting is the result for women, which make more trips in a day (figure 2) than men, but make shorter daily distance (figure 3), so their individual trips must be quite shorter in average than men's. 


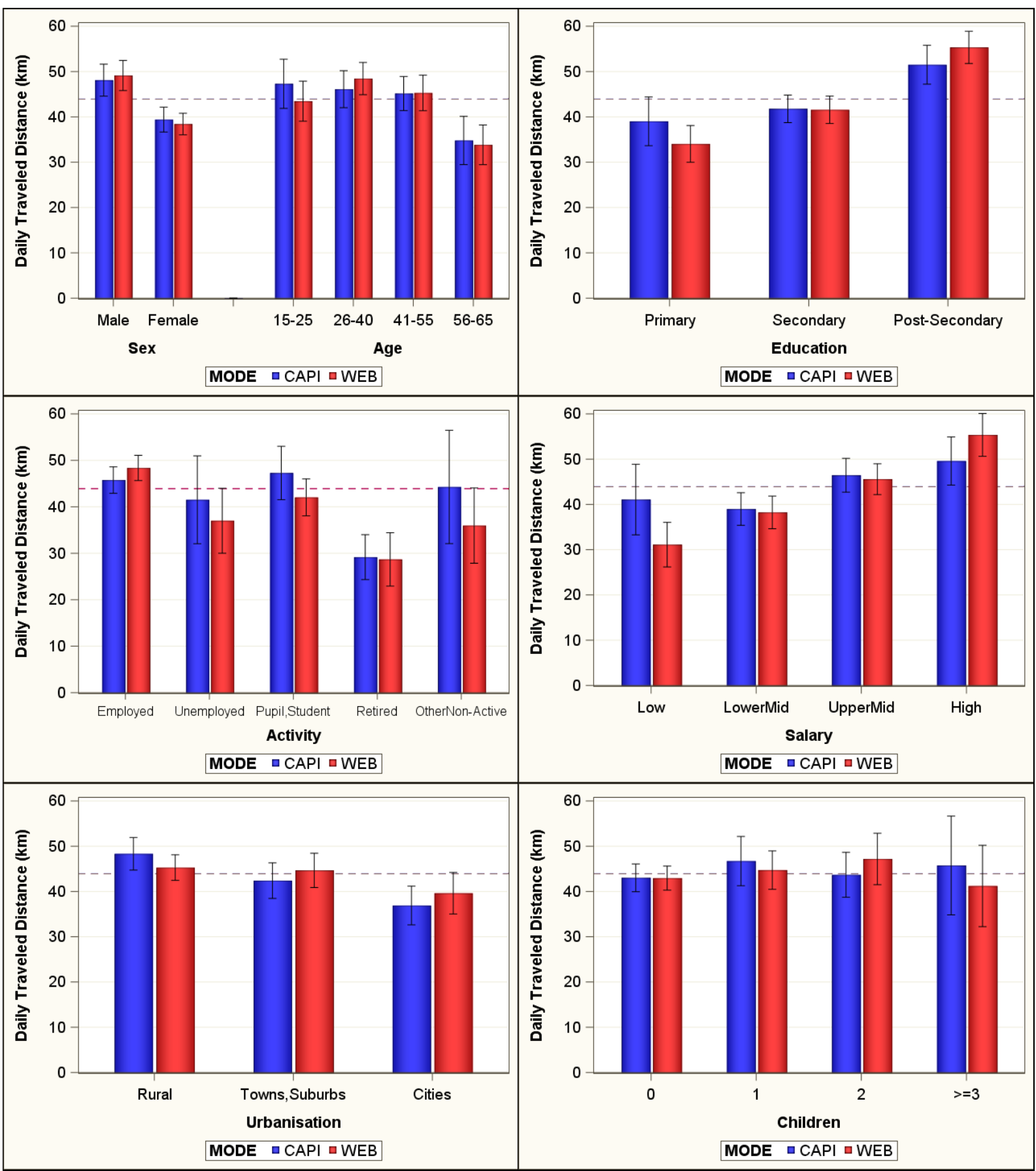

Figure 3 Daily travelled distance per person by: sex, age (upper left), education (upper right), activity status (middle left), salary level (middle right), degree of urbanisation (lower left) and number of children in a household (lower right).

Source: SURS, 2020.

\section{Mode Measurement Effect}

To be able to say if the differences in estimates can be attributed to mode with some statistical confidence, we apply the regression models to the same 2 key statistics, (1) the average number of trips and (2) daily distance.

\section{Number of Trips}

The number of trips is a count data variable, therefore, a dispersed Poisson model is used for its distribution and run with the generalized linear regression model. We 
control the regression for the same socio-demographic variables as presented in the previous estimates diagrams (figure 2 and figure 3). These are 4 basic sociodemographics: sex, age, education and activity status; and 3 additional variables: salary level, a degree of urbanisation and a number of children in a household. Additionally, we included 2 control variables collected by the questionnaire. One is the type of day, be it a workday (Monday to Friday) or weekend (Saturday and Sunday). The other one is a degree of internet usage, which is particularly good control variable in our case, because it might measure the WEB respondent's errors due to the level of internet proficiency. 4 categories of internet usage are: 'regular', 'last time use was 3 months ago', or 'year ago' and 'being without internet'. Data show, that not all WEB respondents fall into the 'regular' category and some even reported 'no internet', so they must have completed the questionnaire elsewhere and/or with help.

Table 4 shows the regression results for average number of trips per trip-maker in a day. The reference class includes the following: Male, Aged 26-40, Secondary education, Employed, Lower-Middle salary, middle degree of urbanisation (Towns, Suburbs), 0 children, Workday and Regular internet user.

Table 4 Regression results for average number of trips

\begin{tabular}{|c|c|c|c|c|c|c|}
\hline Category & Coefficients & Estimate & Std. Error & t value & $\operatorname{Pr}(>|\dagger|)$ & \\
\hline & (Intercept) & 1.151 & 0.023 & 51.1 & $<2 \mathrm{e}-16$ & $* * *$ \\
\hline Sex & Female & 0.038 & 0.013 & 2.9 & 0.00390 & $* *$ \\
\hline \multirow[t]{3}{*}{ Age } & $15-25$ & -0.038 & 0.034 & -1.1 & 0.25439 & \\
\hline & $41-55$ & 0.012 & 0.016 & 0.7 & 0.46639 & \\
\hline & $56-65$ & 0.004 & 0.025 & 0.1 & 0.88524 & \\
\hline \multirow[t]{2}{*}{ Education } & Primary & -0.083 & 0.021 & -4.0 & $7.6 e-05$ & $* * *$ \\
\hline & Post-Secondary & 0.110 & 0.016 & 7.0 & $2.5 e-12$ & $* * *$ \\
\hline \multirow[t]{4}{*}{ Activity } & Unemployed & 0.014 & 0.027 & 0.5 & 0.58626 & \\
\hline & Pupil, Student & 0.016 & 0.037 & 0.4 & 0.67136 & \\
\hline & Retired & -0.003 & 0.030 & -0.1 & 0.91679 & \\
\hline & Non-Active & -0.001 & 0.027 & 0.0 & 0.98164 & \\
\hline \multirow[t]{3}{*}{ Salary } & Low & -0.087 & 0.025 & -3.4 & 0.00059 & $* * *$ \\
\hline & Upper Middle & 0.053 & 0.016 & 3.4 & 0.00077 & *** \\
\hline & High & 0.091 & 0.018 & 4.9 & $9.5 e-07$ & $* * *$ \\
\hline \multirow[t]{2}{*}{ Urbanisation } & Rural & -0.017 & 0.015 & -1.1 & 0.25083 & \\
\hline & Cities & 0.070 & 0.018 & 4.0 & $7.3 e-05$ & $* * *$ \\
\hline \multirow[t]{3}{*}{ Children } & 1 & 0.064 & 0.017 & 3.7 & 0.00019 & $* * *$ \\
\hline & 2 & 0.086 & 0.019 & 4.5 & $7.9 e-06$ & $* * *$ \\
\hline & $>=3$ & 0.134 & 0.035 & 3.9 & 0.00012 & $* * *$ \\
\hline Type of Day & Weekend & -0.217 & 0.015 & -14.1 & $<2 \mathrm{e}-16$ & $* * *$ \\
\hline \multirow{3}{*}{ Internet Use } & 3 Months Ago & -0.298 & 0.078 & -3.8 & 0.00013 & $* * *$ \\
\hline & 1 Year Ago & -0.154 & 0.041 & -3.8 & 0.00017 & $* * *$ \\
\hline & No Internet & -0.117 & 0.040 & -2.9 & 0.00368 & ** \\
\hline MODE & WEB & -0.061 & 0.013 & -4.6 & $5.4 \mathrm{e}-06$ & $* * *$ \\
\hline
\end{tabular}

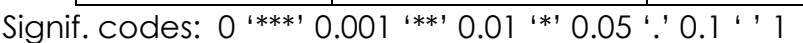

Dispersion parameter for quasipoisson family taken to be 155.3288

Null deviance: 981641 on 6215 degrees of freedom

Residual deviance: 882721 on 6192 degrees of freedom

Source: SURS, 2020.

The results in table 4 show, that any age and activity classes and the rural urbanisation class have no statistically significant effect. All other classes of variables do have a significant effect, including the mode (see the class MODE=WEB at the bottom of table 4). The exponential of the coefficient for WEB mode class gives the 
rate of the effect, which contributes $-5.9 \%$. This means that, controlling for the effect of socio-demographic and other auxiliary variables, person responding in WEB mode reports on average $5.9 \%$ less trips compared to CAPI mode. $95 \%$ confidence interval for the mode effect estimate is between $3.4 \%$ and $8.3 \%$ ( $p$ value $=5.4 \cdot 10^{-6}$ ). We can say that the mode measurement effect is identified in the number of trip statistic.

We can see that for the significant variables in table 4 (which excludes age and activity status) the levels and signs of regression coefficients correspond to the differences of estimates in figure 2. However, despite there are some apparent similarities, the regression results in table 4 cannot be directly compared to the above figure 2 due to the different analyses. To see the connection between the two methods (the graphical comparison in figure 2 and the regression analysis in table 4), we can think of the following regression model. The simpler Poisson regression model for the number of trips that would include only the covariates sex and mode and the interaction between them, would give us the same estimates as in the first diagram (upper left) in figure 2 comparing only the first four bars for sex category. The interaction would be needed to explain the smaller difference between modes for women compared to the difference between modes for men. The same is true for any compared category in figure 2, if we substitute the covariate sex with the corresponding category in question. However, we would have obtained from the regression model the information about the significance of the partial effect of mode on the estimate, that is, the effect left after controlling for differences in sex. Such simpler model would be just a special case of the regression analysis in table 4. The full regression analysis is thus more complete, as it controls for many possible influences at the same time.

\section{Daily Distance}

For the daily distance statistic we use an OLS regression model on the logarithm of distance. Due to the logarithm, the regression coefficients do not directly represent the estimates' results. The same control variables and same classes including the reference one, are used as in the above regression for the number of trips.

Table 5 shows the regression results for daily distance. By comparing the results to the case of the number of trips (table 4), we can see, that in this case some additional variables lose its statistical significance. These variables are: number of children, type of day and 2 classes of internet usage (table 5). We can see in table 5, that in this case, the mode class (MODE=WEB) shows no statistically significant effect on the daily distance estimate.

\section{Discussion}

Let us summarise the above regression results. The mode measurement effect persists in the estimate of the number of trips (WEB mode gives less trips). On the other hand, mode has no significant measurement effect on the estimate of daily travelled distance. Because the overall daily distance is similar to CAPI and WEB mode, we can conclude that the WEB respondents are really inclined to join two or more trips into one reported, with summed up distances of possible individual trips.

However, if we recall the aforementioned experiences from data editing process, we see that a lot of such cases (joined trips) have been considered during the data correction. The observed persisting mode difference in the regression analysis tells us, that the editing process had not covered such cases completely. Indeed, despite many manual corrections, it was sometimes impossible to reconstruct the possible correct trips. We can therefore conclude that the mode measurement effect observed in the estimate of the number of trips has actually two consecutive 
sources. First, WEB respondents report less trips by joining more trips into one single, and second, despite all the efforts put into the questionnaire design and the correction controls, the techniques for controlling and correcting such complex questionnaire responses were still insufficient to correct for this particular measurement error.

Table 5 Regression results for daily travelled distance

\begin{tabular}{|c|c|c|c|c|c|c|}
\hline Category & Coefficients & Estimate & Std. Error & † value & $\operatorname{Pr}(>|\dagger|)$ & \\
\hline & (Intercept) & 3.106 & 0.057 & 54.6 & $<2 \mathrm{e}-16$ & *** \\
\hline Sex & Female & -0.268 & 0.033 & -8.1 & $7.0 e-16$ & $* * *$ \\
\hline \multirow[t]{3}{*}{ Age } & $15-25$ & 0.067 & 0.083 & 0.8 & 0.42078 & \\
\hline & $41-55$ & -0.004 & 0.042 & -0.1 & 0.91938 & \\
\hline & $56-65$ & -0.217 & 0.063 & -3.4 & 0.00060 & *** \\
\hline \multirow[t]{2}{*}{ Education } & Primary & -0.179 & 0.050 & -3.6 & 0.00037 & *** \\
\hline & Post-Secondary & 0.264 & 0.041 & 6.4 & $1.3 e-10$ & *** \\
\hline \multirow[t]{4}{*}{ Activity } & Unemployed & -0.124 & 0.067 & -1.9 & 0.06329 & . \\
\hline & Pupil, Student & 0.074 & 0.092 & 0.8 & 0.42017 & \\
\hline & Retired & -0.168 & 0.073 & -2.3 & 0.02180 & * \\
\hline & Non-Active & -0.128 & 0.066 & -1.9 & 0.05258 & \\
\hline \multirow[t]{3}{*}{ Salary } & Low & -0.033 & 0.060 & -0.5 & 0.58454 & \\
\hline & Upper Middle & 0.120 & 0.040 & 3.0 & 0.00253 & ** \\
\hline & High & 0.249 & 0.047 & 5.2 & $1.6 \mathrm{e}-07$ & *** \\
\hline \multirow[t]{2}{*}{ Urbanisation } & Rural & 0.188 & 0.037 & 5.1 & $3.1 e-07$ & *** \\
\hline & Cities & -0.136 & 0.045 & -3.0 & 0.00284 & ** \\
\hline \multirow[t]{3}{*}{ Children } & 1 & -0.006 & 0.044 & -0.1 & 0.89076 & \\
\hline & 2 & -0.006 & 0.050 & -0.1 & 0.90838 & \\
\hline & $>=3$ & 0.034 & 0.092 & 0.4 & 0.71042 & \\
\hline Type of Day & Weekend & -0.033 & 0.037 & -0.9 & 0.37111 & \\
\hline \multirow[t]{3}{*}{ Internet Use } & 3 Months Ago & -0.248 & 0.162 & -1.5 & 0.12632 & \\
\hline & 1 Year Ago & -0.108 & 0.092 & -1.2 & 0.24124 & \\
\hline & No Internet & -0.192 & 0.095 & -2.0 & 0.04232 & $*$ \\
\hline MODE & WEB & -0.033 & 0.034 & -1.0 & 0.33289 & \\
\hline
\end{tabular}

Signif. codes: 0 '***' 0.001 '**' 0.01 '*' 0.05 '.' 0.1 ' ' 1

Residual standard error: 17.65 on 6192 degrees of freedom

Multiple R-squared: 0.0594, Adjusted R-squared: 0.0559

F-statistic: 16.99 on 23 and 6192 DF, p-value: $<2.2 \mathrm{e}-16$

Source: SURS, 2020.

\section{Conclusion}

The objective of this study was the analysis of the possible mode measurement effect in the key statistic estimates in the daily passenger mobility survey. The results of the analysis show that the mode measurement effect persist in the estimate of the number of daily passenger's trips in WEB mode, whereas the daily distance stays similar between the CAPI and WEB mode. The conclusion is that the WEB respondents are inclined to join two or more trips into one single trip. This result is similar to the one reported in other similar surveys (Bayart, Bonnel, 2015). On the other hand, we did not observe (at least on the aggregate level) that the WEB respondents would be inclined to avoid some less important trips, because there is no significant difference in the overall daily distance. However, the detailed analysis on the transport mode would probably give us clearer picture, but was beyond the scope of this study.

We can say that the observed measurement error has two consecutive sources. On one hand, the questionnaire content is quite demanding, and the idea and the 
concept of the trip is not easy to grasp and understand. This is the general reason for increased error rate particularly in WEB (self-completion) mode. It is however a bit of a puzzle, why the WEB respondents report joined trips. One possibility is that they join trips in order to go through the questionnaire more quickly (e.g. if respondent did many trips in a day, the motivation to fill in all trips may drop). Whereas in CAPI mode, the interviewer can remind the respondent and intervene to correct the description of the trip or to name additional trips. On the other hand, the complex data editing controls were still insufficient to correct completely for such measurement error. We have learned that the geocoding would be of great help in the similar future surveys, since it avoids the errors already in the questionnaire completion stage and possibly reduces also the respondent's burden. Moreover, the data editing process would be accordingly less complex and the manual corrections could be completely avoided. However, the problem with geocoding usage might be in the rising public sensitivity regarding the revealing detailed information on the individual's precise movements (tracking time and location).

Future research of measurement effect in the presented survey should focus also on more detailed parameter analyses, such as the transport mode and the purpose of a trip. Considering the data quality, the usage of methods for statistically correcting for mode measurement effect may be beneficial.

\section{References}

1. Arnež, M., Belak, E., Bučar, N., Nemec, M., Rutar, K., Zupanc, L. (2018). Transition to WEB data collection in household surveys at SURS - what have we learned so far. Q2018 European Conference on Quality in Official Statistics, Kraków, Poland, 26-29 June 2018.

2. Bayart, C., Bonnel, P. (2010). The mixing of survey modes: application to Lyon web and face-to-face household travel survey. 2th World Conference on Transportation Research, Lisbon, 11-15 July 2010.

3. Bayart, C., Bonnel, P. (2015). How to Combine Survey Media (Web, Telephone, Face-toFace): Lyon and Rhône-alps Case Study. Transportation Research Procedia, Vol. 11, pp. 118-135.

4. Berzelak, J. (2014). Mode effects in web surveys. Ph.D. dissertation, Faculty for social sciences, University of Ljubljana, Ljubljana.

5. Bonnel, P., Bayart, C., Smith, B. (2015). Workshop Synthesis: Comparing and Combining Survey Modes. Transportation Research Procedia, Vol. 11, pp. 108-117.

6. Bučar, N., Belak, E., Arnež, M., Rutar, K. (2018). Nonresponse in Web Surveys in Official Statistics. International Workshop on Household Survey Nonresponse, Budapest, Hungary, 22-24 August 2018.

7. Center for Transport Analytics (2019). The Danish National Travel Survey. Available at https://www.cta.man.dtu.dk/english/national-travel-survey [28 October 2020].

8. De Leeuw, E. (2018). Mixed-mode: Past, present, and future. Survey Research Methods, Vol. 12, No. 2, pp. 75-89.

9. Eurostat (2018), Eurostat Guidelines on Passenger Mobility Statistics. Eurostat, Luxembourg.

10.Hox, J., de Leeuw, E., Klausch, T. (2017). Mixed mode research: issues in design and analysis. In Total survey error in practice, Biemer, P. et al. (Eds.), Wiley, Hoboken, NJ, pp. $511-530$.

11.Klausch, T. (2014). Informed design of mixed-mode surveys: evaluating mode effects on measurement and selection error. Ph.D. Dissertation, Utrecht University. Available at https://dspace.library.uu.nl/handle/1874/300673 [26 November 2020].

12.Martin, P., Lynn, P. (2011). The Effects of Mixed Mode Survey Designs on Simple and Complex Analyses. ISER Working Paper Series, No. 2011-28, ISER, University of Essex, Colchester. Available at https://EconPapers.repec.org/RePEc:ese:iserwp:2011-28 [26 November 2020].

13.Škafar, A. (2018). Passenger mobility and road traffic. Final Report. SURS, Ljubljana, Slovenia. 
14.Škafar, A. (2019a). Methodological Explanation on Daily Passenger Mobility. SURS, Ljubljana, Slovenia.

15.Škafar, A. (2019b). Standardno poročilo o kakovosti za raziskovanje Dnevna mobilnost potnikov (2017). Quality Report, SURS, Ljubljana, Slovenia.

16.Statistics Netherlands (2016). Transport and mobility 2016. Statistics Netherlands, The Hague/Heerlen/Bonaire.

17.Transport Analysis (2019). The Swedish National Travel survey. Available at https://www.trafa.se/en/travel-survey/travel-survey [28 October 2020].

\section{About the authors}

Vasilij Centrih works at Statistical Office of the Republic of Slovenia at the Department of General Methodology. He is involved in statistical data processing and is particularly responsible for data aggregation and tabulation of several individual surveys as well as the regular statistical production. Author can be contacted at vasilij.centrih@gov.si.

Andrej Viršček is a methodologist at the Statistical office of the Republic of Slovenia. He holds a master's degree in Applied Statistics from University of Ljubljana and a bachelor degree in Business Economics from FELU. He worked as a data journalist at Television Slovenia as a member of award winning team Ekstravisor and as a data scientist in market research company Valicon. Author can be contacted at andrej.virscek@gov.si.

Andreja Smukavec has been working at Statistical office of Slovenia since 2004. She has been the head of General Methodology Department, which is responsible for sampling, editing, sampling errors calculation, modelling, time series analysis and statistical disclosure control, since 2014. She was the leader of the project team, which build the application for standard metadata procedures for aggregation (including sampling errors calculation), tabulation and disclosure control in the years 2013 - 2016. Andreja Smukavec is active in international area, especially in the field of statistical confidentiality, she is a member of Working Group on Methodology and Expert Group on SDC at European level. Author can be contacted at andreja.smukavec@gov.si.

Nataša Bučar works at the Statistical Office of the Republic of Slovenia since 2017. She works in area of sampling and weighting social and business surveys at the Department of General Methodology. Author can be contacted at natasa.bucar2@gov.si.

Marta Arnež works at Statistical Office of Slovenia at the Department of General Methodology. She works in the field of survey methodology in surveys of persons and households (sampling, weighting, non-response analysis). Author can be contacted at marta.arnez@gov.si. 